\title{
Dermal Injection of Immunocytes Induces Psoriasis
}

Tamara Wrone-Smith* and Brian J. Nickoloff* ${ }^{\star \ddagger}$

$*$ Department of Pathology, University of Michigan, Ann Arbor, Michigan 48104; and ${ }^{\ddagger}$ Department of Pathology, Loyola University

School of Medicine, Maywood, Illinois 60153

\begin{abstract}
Establishing direct and causal relationships among the confederacy of activated cell types present in psoriasis has been hampered by lack of an animal model. Within psoriatic plaques there are hyperplastic keratinocytes, infiltrating immunocytes, and activated endothelial cells. The purpose of this study was to determine if psoriasis is primarily a disorder of keratinocytes or the immune system. Using a newly developed experimental system in which full-thickness human skin is orthotopically transferred onto severe combined immunodeficient mice, autologous immunocytes were injected into dermis, and the resultant phenotype characterized by clinical, histologic, and immunophenotypic analyses. Engraftment of samples included both uninvolved/ symptomless (PN) skin removed from patients with psoriasis elsewhere, or from healthy individuals with no skin disease (NN skin). In 10 different experiments involving 6 different psoriasis patients, every PN skin was converted to a full-fledged psoriatic plaque skin by injection of autologous blood-derived immunocytes. In all but one psoriatic patient, the immunocytes required preactivation with IL-2 and superantigens to convert PN skin into psoriatic plaque skin. In every case, resultant plaques were characterized by visible presence of flaking and thickened skin, loss of the granular cell layer, prominent elongation of rete pegs with a dermal angiogenic tissue reaction, and infiltration within the epidermis by $\mathrm{T}$ cells. Lesional skin displayed 20 different antigenic determinants of the psoriatic phenotype. None of the four NN skin samples injected with autologous immunocytes converted to psoriatic plaques. We conclude that psoriasis is caused primarily by the ability of pathogenetic blood-derived immunocytes to induce secondary activation and disordered growth of endogenous cutaneous cells including keratinocytes and vascular endothelium. (J. Clin. Invest. 1996. 98:1878-1887.) Key words: psoriasis • T cells • severe combined immunodeficient mice $\bullet$ superantigen $\bullet$ antigen-presenting cell
\end{abstract}

\section{Introduction}

Psoriasis is a common and enigmatic chronic skin disease that can be genetically transmitted with phenotypic expression pri-

Address correspondence to Dr. Brian J. Nickoloff, Loyola University Medical Center, Oncology Institute - Bldg. 112, Room 301, 2160 S. First Ave., Maywood, Illinois 60153. Phone: 708-327-3241; FAX: 708327-3239; E-mail: bnickol@luc.edu

Received for publication 8 April 1996 and accepted in revised form 15 August 1996.

J. Clin. Invest.

(C) The American Society for Clinical Investigation, Inc. 0021-9738/96/10/1878/10 \$2.00

Volume 98, Number 8, October 1996, 1878-1887 marily confined to skin (1). Clinically, psoriasis is considered a disease of the entire skin, with the most common presentation being well circumscribed erythematous scaling plaques that may be symmetrically distributed (2). There are several histological abnormalities of acute/chronic lesions including: marked epidermal keratinocyte hyperplasia with altered differentiation and elongated rete pegs; tissue inflammation with neutrophils in the stratum corneum; influx of immunocytes including both CD4 and CD8 positive lymphocytes and dendritic antigen presenting cells (APCs); and endothelial cell activation (3). Despite several decades of intensive research, a major unresolved issue in contemporary skin biology is whether the genetically-transmitted defect in psoriasis resides fundamentally in the indigenous epidermal keratinocyte, or the recruited circulatory immunocyte (3). This problem is made more complex by the recent recognition that keratinocytes can also function as accessory cells in various immunological reactions (4). Another obstacle in the quest for understanding this complicated cutaneous disorder has been the absence of appropriate tissue or cell culture model systems in vitro, or animal models with spontaneous or inducible disease, that express all the aforementioned histological abnormalities.

Indirect experimental evidence has been interpreted as supporting both a primary defect in keratinocytes (5-8), as well as in immunocytes (9-19). To directly address whether keratinocyte hyperplasia and altered differentiation is a cause or consequence of abnormal immunocyte activity, we developed an experimental model system in which keratome skin samples from normal volunteers and psoriatic patients are grafted onto severe combined immunodeficient (SCID) ${ }^{1}$ mice (20).

We reasoned that if psoriasis was fundamentally a disease of the immune system, then injection of blood-derived immunocytes should be necessary and sufficient to produce conversion of symptomless (PN) skin into full-fledged psoriatic plaque (PP) skin. This report documents the clinical, histological, and immunophenotypic features of transplanted human skin obtained from normal volunteers (NN skin) and psoriatic patients injected with autologous blood-derived immunocytes. Based on the results using this SCID mouse model, we conclude that psoriasis is mediated by immunocytes derived from the circulation and once activated and present in skin, the immunocompetent cells secondarily induce keratinocyte and endothelial cell proliferation.

\section{Methods}

Patients. PN skin was biopsied $(6 \times 2 \times 0.05 \mathrm{~cm}$ keratome containing both dermis and epidermis) from psoriatic patients $(n=6)$ after informed consent was obtained and the study approved by the University of Michigan Human Subjects Committee (Ann Arbor, MI).

1. Abbreviations used in this paper: NN, no skin disease; PCNA, proliferating cell nuclear antigen; $\mathrm{PN}$, symptomless; $\mathrm{PP}$, psoriatic plaque; SCID, severe combined immunodeficient. 
Keratome biopsies were obtained from clinically symptomless skin on the lower back or buttocks. The psoriatic patients included two females and four males (aged 28-65). Normal full thickness human skin $(n=4)$ was obtained from healthy adult donors (NN skin, aged 33-57) having no family history or clinical evidence of psoriasis.

Human skin/SCID mouse chimera and tissue processing. Human skin xenografts were orthotopically transplanted onto 7-8-wk-old SCID mice (Taconic Farms Inc., Germantown, NY) following previously described procedures (20). Briefly, human skin xenografts (measuring $1.5 \times 1.5 \times 0.5 \mathrm{~cm}$ ) were sutured to the flank area of each SCID mouse with absorbable 5-0 Vicryl Rapide suture (Ethicon, Somerville, NJ) and covered with Xeroform dressings (Kendall Co., Mansfield, MA). Dressings were removed 1 wk later and animals were maintained pathogen-free throughout the study. 2-3 wk after transplantation, autologous cells or reagents diluted in sterile PBS were injected intradermally ( $300 \mu \mathrm{lol})$ into the xenograft. Human skin/SCID mouse chimeras were killed by $\mathrm{CO}_{2}$ asphyxiation within 2-3 wk of the last intradermal injection, and 4-mm punch biopsies (Baker's Biopsy Punch; Cummins Derm, Miami, FL) were obtained from each xenograft. Biopsies were fixed in 10\% neutral-buffered formalin for paraffin embedding, and/or mounted on gum tragacanth (Sigma Chemical Co., St. Louis, MO) snap frozen in liquid nitrogenchilled isopentane, and stored at $-80^{\circ} \mathrm{C}$.

Immunohistochemistry of human skin grafts. 5- $\mu \mathrm{m}$ thick cryostat tissue sections were acetone fixed or 3- $\mu \mathrm{m}$ formalin fixed, paraffinembedded samples were dewaxed in xylene and ethanol and rehydrated. For PCNA immunostaining, paraffin-embedded samples were additionally microwaved in $0.01 \mathrm{M}$ citrate buffer, $\mathrm{pH}$ 6.0, to optimize antigen retrieval. Sections were immunostained to detect a variety of antigens using a highly sensitive biotin-avidin immunoperoxidase technique (Vectastain kit; Vector Laboratories, Inc., Burlingame, CA) as previously described (20). The panel of antibodies used is summarized in Table I.

Reagents. Various stimuli injected into the grafts included: PBS (control); IFN- $\gamma(1 \mu \mathrm{g}$, Genentech Inc., South San Francisco, CA); GM-CSF (400 ng, R \& D Systems, Inc., Minneapolis, MN); LPS (1 $\mu \mathrm{g}$, Sigma Chemical Co.); and keratinocyte growth factor (KGF; 500 ng, R \& D Systems, Inc.).

Cell culture studies. Autologous immunocytes were isolated from heparinized blood by Ficoll-Hypaque ${ }^{\circledR}$ (Pharmacia LKB Biotechnology Inc., Piscataway, NJ) density centrifugation. 1-2 $\times 10^{6}$ $\mathrm{PBMC} / \mathrm{ml}$ were cultured on tissue culture dishes (Corning Glass Works, Corning, NY) in complete media containing $10 \%$ heat-inactivated autologous serum in RPMI-1640 containing $25 \mathrm{mM}$ Hepes (GIBCO BRL, Gaithersberg, MD) supplemented with $2 \mathrm{mM}$ L-glutamine, 100 $\mathrm{U} / \mathrm{ml}$ penicillin, $100 \mu \mathrm{g} / \mathrm{ml}$ streptomycin, and $50 \mu \mathrm{g} / \mathrm{ml}$ gentamicin (GIBCO BRL). Immunocytes were cultured in complete media with or without $1 \mu \mathrm{g} / \mathrm{ml}$ each of Staphylococcal enterotoxins (SE) SEB and SEC2 (Toxin Technologies, Sarasota, FL) and $20 \mathrm{U} / \mathrm{ml}$ human IL-2 (Boehringer Mannheim Biochemicals, Indianapolis, IN) for $48 \mathrm{~h}$ at $37^{\circ} \mathrm{C}$ in a humidified atmosphere containing $5 \% \mathrm{CO}_{2}$. After incubation cells were removed from the plates, washed twice in RPMI-1640 and resuspended in sterile PBS.

Pathological assessment. Biopsies for histological evaluation included 4-mm punch biopsies obtained from human skin grafted onto SCID mice. The determination of mean epidermal thickness was obtained by first defining a standard length of epidermis (1.3 microns), and then measuring epidermal area using IP lab spectrum image analysis software (Signal Analytics Corp.), and then dividing by the length. Results portrayed are derived from all 10 subjects, 6 psoriatic patients and 4 healthy control subjects, and for epidermal thickness measurements represent the mean values determined by duplicate image analyses. The other results portrayed are representative examples from all these 10 subjects. All values were subjected to statistical evaluation using Students $t$ test and considered significant if $P<0.05$.

\section{Results}

Response of PN skin to soluble mediators of inflammatory and immunologic reactions. After engraftment onto SCID mice, PN skin clinically appears tan/brown with occasional thin

Table I. Panel of Antibodies Used for Immunostaining

\begin{tabular}{|c|c|c|}
\hline $\begin{array}{c}\text { Antigen } \\
\text { (specificity) }\end{array}$ & $\begin{array}{l}\text { Antibodies } \\
\text { (isotype) }\end{array}$ & Source \\
\hline \multicolumn{3}{|l|}{ T cells } \\
\hline CD3 & Leu-4 (IgG1) & Becton Dickinson (Mountain View, CA) \\
\hline $\mathrm{CD} 4$ & Leu-3a (IgG1) & Becton Dickinson \\
\hline CD8 & Leu-2a (IgG1) & Becton Dickinson \\
\hline CD25/IL-2 receptor & TAC (IgG2a) & Immunotech (Westbrook, ME) \\
\hline CD45RO & UCHL-1 (IgG2a) & DAKO Corp. (Carpinteria, CA) \\
\hline \multicolumn{3}{|l|}{ Adhesion molecules } \\
\hline CD54/ICAM-1 & RR 1/1 (IgG1) & Genzyme Corp. (Cambridge, MA) \\
\hline CD106/VCAM-1 & $51-10 \mathrm{C} 9(\mathrm{IgG} 1)$ & Pharmingen (San Diego, CA) \\
\hline CD62E/E-selectin & $(\mathrm{IgG} 1)$ & Genzyme Corp. \\
\hline CLA & HECA-452 (rat IgM) & L. Picker (20) \\
\hline \multicolumn{3}{|l|}{ Miscellaneous } \\
\hline CD29/ß1 integrin & 4B4 (IgG1) & Coulter (Hialeah, FL) \\
\hline CD1a & Leu-6 (IgG2b) & Becton Dickinson \\
\hline $\mathrm{CD} 14$ & Leu-M3 (IgG1) & Becton Dickinson \\
\hline CD80 & B7-1 (IgG1) & Pharmingen \\
\hline Factor XIIIa & (Rabbit polyclonal) & Calbiochem (La Jolla, CA) \\
\hline HLA-DR & L243 (IgG2a) & Becton Dickinson \\
\hline Involucrin & (Rabbit polyclonal) & Biomedical Technologies Inc. (Stoughton, MA) \\
\hline Keratin 16 & K8.12 (IgG1) & Sigma Chemical Co. (St. Louis, MO) \\
\hline MAC 387 & $(\mathrm{IgG} 1)$ & DAKO Corp. \\
\hline PCNA & $\mathrm{PC} 10(\operatorname{IgG} 2 \mathrm{a})$ & DAKO Corp. \\
\hline
\end{tabular}


scale, and has a mean epidermal thickness of $97 \pm 12 \mu \mathrm{m}$. Histologically there is a compact stratum corneum and intact granular cell layer, with occasional lymphocytes in the superficial vascular plexus as previously described (20). There was no significant change observed in either the clinical or histological (Fig. $1 A$ ) appearance of PN skin injected with phosphate buffered saline (PBS). To determine how transplanted PN skin would respond to various stimuli implicated in the cytokine network within psoriatic plaques (3), intradermal injection was performed after engraftment. After exposure to these soluble mediators, there was variable clinical change that included induction of slight erythema, scale, and thickening of the skin. Representative routine light microscopic features of PN skin are portrayed after injection with IFN- $\gamma$ plus GM-CSF (16) (Fig. $1 \mathrm{~B}$ ), and the 48-h conditioned medium from immunocytes activated by IL-2 plus bacterial-derived superantigens SEB and SEC 2 (Fig. $1 C$ ). Activating reagents were selected because of their ability to induce $\mathrm{T}$ cell expression of skin selective homing receptor (CLA), and the potential relevance of bacterial superantigens to psoriasis $(3,21,22)$. There was a more conspicuous appearance of lymphocytes in the PN skin injected with the conditioned medium (Fig. $1 \mathrm{C}$ ) compared with the other stimuli.

Varying degrees of epidermal keratinocyte proliferation could be induced in this model system depending on the type of soluble mediator injected into the dermis (Table II). The largest increase in epidermal thickness followed injection of conditioned medium from autologous psoriatic blood-derived immunocytes pre-activated with IL-2/SEB/SEC2. While this increased epidermal thickness was the only result with statistical significance $(P<0.05)$ compared to PBS amongst the other soluble mediators listed in Table II, this value $(239 \pm 38 \mu \mathrm{m})$ did not represent a significant change when compared to the increase observed using IL-2/SEB/SEC2 alone (202 $\pm 24 \mu \mathrm{m})$.

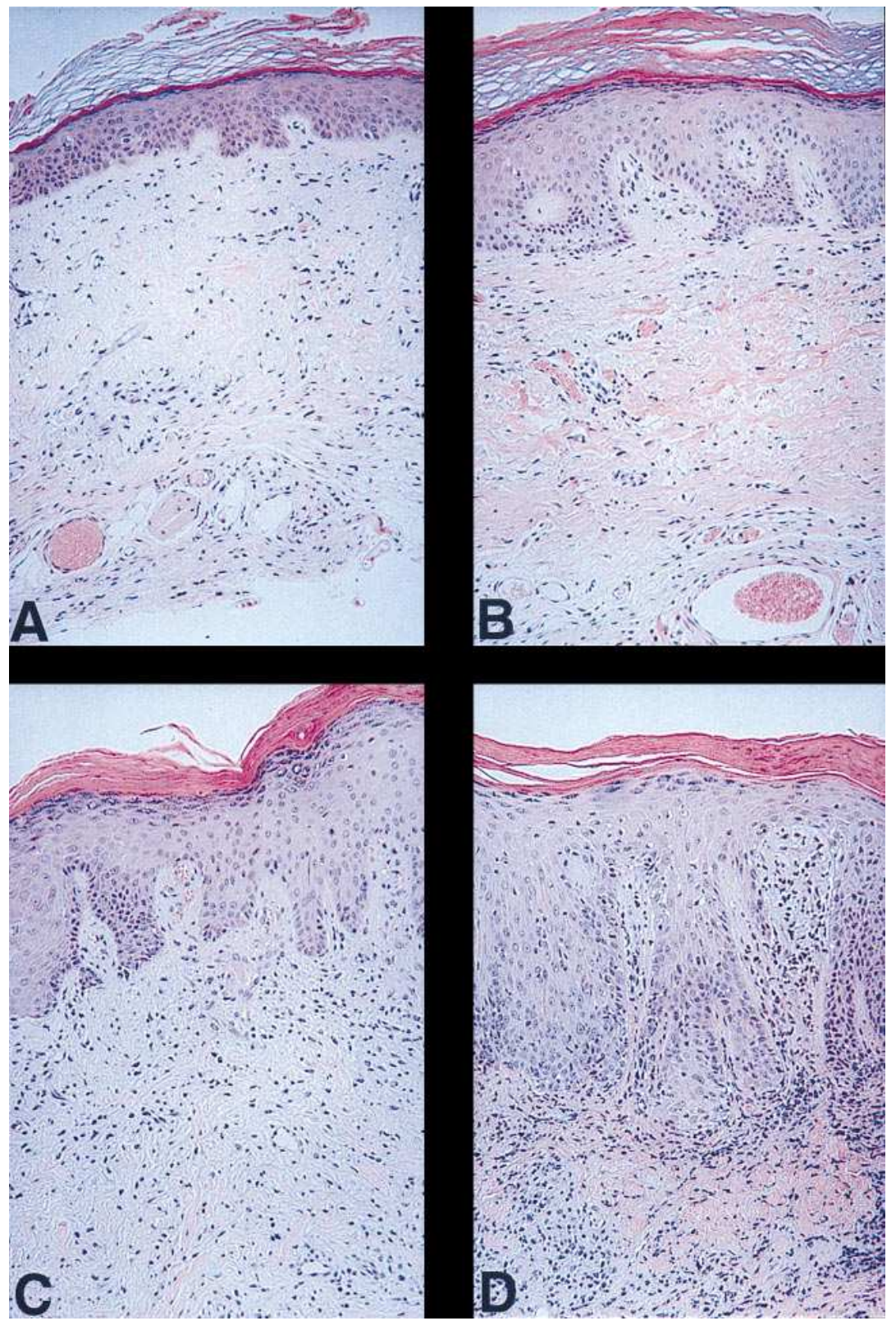

Figure 1. Light microscopic appearance of transplanted PN skin injected with PBS $(A)$; IFN- $\gamma$ plus GM-CSF $(B)$; conditioned medium obtained from activated autologous immunocytes $(C) ; 3 \times 10^{6}$ activated autologous immunocytes $(D)$. Note the slight thickening of skin after injection of IFN- $\gamma$ plus GM-CSF compared to the PBS injected skin. After injection of the conditioned medium there is compacted stratum corneum, mild acanthosis, with only focal loss of the granular cell layer. In contrast, after injection of autologous activated immunocytes, there is confluent parakeratotic scale, complete absence of a granular cell layer, prominent acanthosis with elongation of rete pegs, and a mononuclear cell infiltrate in the dermis and epidermis. Hematoxylin-eosin stain; $\times 80$. 
Table II. Epidermal Thickness of PN Skin Following Injection with Various Stimuli

\begin{tabular}{lc}
\hline \multicolumn{1}{c}{ Stimulus/dose } & Epidermal thickness \\
\hline PBS & $118 \pm 21$ \\
LPS $(1 \mu \mathrm{g})$ & $149 \pm 27$ \\
GM-CSF $(400 \mathrm{ng})$ & $155 \pm 29$ \\
IFN- $\gamma(1 \mu \mathrm{g})$ & $141 \pm 27$ \\
GM-CSF plus IFN- $\gamma$ & $198 \pm 28$ \\
KGF $(500 \mathrm{ng})$ & $208 \pm 25$ \\
SEA $(1 \mu \mathrm{g})$ plus SEB $(1 \mu \mathrm{g})$ & $182 \pm 22$ \\
IL-2/SEB/SEC2 & $202 \pm 24$ \\
Conditioned medium*-Psoriatic Patients & $239 \pm 38$ \\
Conditioned medium*-Healthy Control Patients & $184 \pm 24$ \\
\hline
\end{tabular}

After engraftment, PN skin was injected with the indicated dose of stimuli (in $300 \mu \mathrm{l}$ ) and the skin harvested and prepared for epidermal thickness measurements (in microns) as described in Methods. The data presented represents mean values from two or three different skin grafts. Despite the increases noted above in epidermal thickness induced by intradermal injection of several different types of soluble mediators, no full-fledged psoriatic plaques were produced. ${ }^{*}$ Conditioned medium was obtained by stimulating Ficoll-Hypaque isolated immunocytes from either psoriatic patients; or from healthy control, non-psoriatic patients; $\left(10^{6}\right.$ cells/ml $)$ for $48 \mathrm{~h}$ using IL-2 $(20 \mathrm{U} / \mathrm{ml})$, SEB $(1 \mu \mathrm{g})$, and SEC2 $(1$ $\mu \mathrm{g})$ in RPMI plus $10 \%$ autologous human serum.

To determine if the conditioned medium of activated psoriatic immunocytes was different from normal control activated immunocytes, conditioned medium from activated allogeneic immunocytes was also tested. Table II reveals that there was no significant difference $(P>0.05)$ between the conditioned medium using activated immunocytes derived from psoriatic $(239 \pm 38 \mu \mathrm{m})$ versus non-psoriatic subjects $(184 \pm 24 \mu \mathrm{m})$. Overall these results suggested that phenotypic changes could be induced in this model system, but because full-fledged psoriatic lesions were not produced using soluble mediators we next investigated the effects of injecting autologous immunocytes directly into transplanted skin grafts (Fig. 1D).

Induction of full fledged psoriatic plaques using immunocytes. To investigate the pathogenicity of immunocytes infiltrating into skin, multiple PN grafts were placed onto SCID mice from six different psoriatic patients. After engraftment, 2-3 $\times 10^{6}$ blood-derived autologous immunocytes were intradermally injected and grafts evaluated 2-4 weeks later as described in Methods (Fig. $1 D$ ). Injected immunocytes were derived from the Ficoll-Hypaque interface, and were cultured for $2 \mathrm{~d}$ either in RPMI plus $10 \%$ autologous human serum (designated unstimulated), or activated by the presence of IL-2 and SEB/SEC2 as described above.

In five out of six psoriatic patients, the injection of nonstimulated autologous immunocytes produced only minimal scale and thickening of skin, with no induction of an angiogenic tissue reaction (Figs. 2, $A$ and $C$ ). However, in one psoriatic patient the unstimulated immunocytes consistently produced striking clinical changes in three different experiments over a 4-mo period of study. These changes were indistinguishable from the other five patients in which their activated immunocytes were injected converting PN skin to PP skin. The changes observed for all six psoriatic patients injected with activated immunocytes included marked induction of scale with thickening of the skin, and a prominent angiogenic tissue reac-
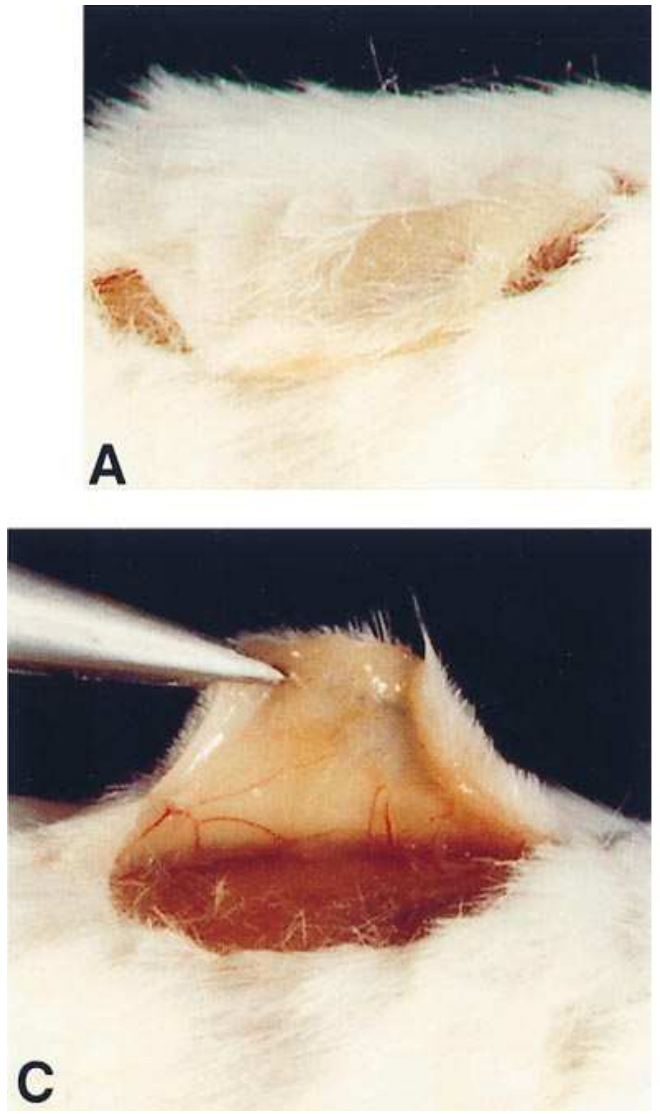
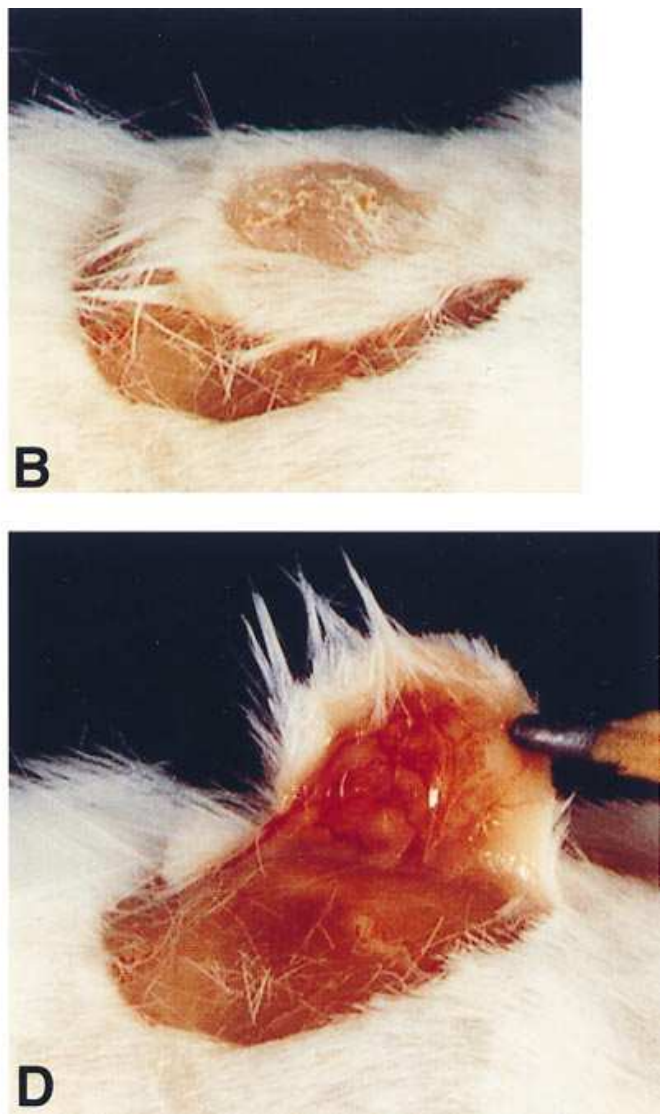

Figure 2. Clinical appearance of transplanted PN skin injected with nonstimulated autologous immunocytes $(A$ and $C)$; or injected with activated autologous immunocytes ( $B$ and $D$ ). Compared with the injection of nonstimulated immunocytes in which the skin is tan, flat, and without scale or angiogenesis ( $A$ and $C$ ), the injection of activated immunocytes produced a thickened, erythematous scaling skin lesion with prominent neo-vascularization of the subcutaneous tissue underlying the human skin graft. 
tion (Figs. 2, $B$ and $D$ ). Interestingly, the patient whose unstimulated immunocytes induced psoriasis had the smallest proportion of her skin involved by the disease (confined to her forearm and elbows) compared with other patients; although her HLA typing revealed she was CW6 positive, which has the highest relative risk for developing psoriasis amongst Caucasians $(23,24)$.

The routine light microscopic appearance of PN skin injected with activated autologous immunocytes was similar amongst all injected grafts and revealed confluent and extensive parakeratotic scale with underlying marked acanthosis, loss of the granular cell layer, and prominent elongation of rete pegs (Figs. $1 D$ and $3 A$ ). There was also abundant lymphocytes seen in the dermis with scattered lymphoid cells in lower and mid-zones of the hyperplastic epidermis. Dermal blood vessels were dilated and occasionally contained murine neutrophils that emigrated from the vasculature to enter the epidermis producing spongiform pustules of Kogoj and Munro's microabscesses; additional hallmarks of psoriatic plaques (Fig. 3 B). The epidermal thickness measurements for PN skin injected with either unstimulated or activated immunocytes is summarized in Fig. 4 (right side). It should be noted that compared to the injection of soluble mediators, the injection of activated immunocytes produced substantially greater changes in the epidermal thickness for all six psoriatic patients studied.

To characterize the response of NN skin (mean epidermal thickness of $91 \pm 14 \mu \mathrm{m}$ ) to dermal injection of either unstimulated or activated immunocytes, four healthy control individuals were biopsied. Following engraftment of NN skin, autologous immunocytes were injected exactly as described for psoriatic patients. In two healthy donors, both unstimulated and activated immunocytes were administered, whereas only activated immunocytes were injected for the other two individuals. However, in all injected NN skin grafts, there was only minimal scale, slight change in the thickness of skin, and no angiogenic tissue response. The histology of injected grafts from all four controls injected with activated autologous immunocytes included mild hyperkeratosis and acanthosis with lymphocytes in the dermis and epidermis, but without the dramatic hyperplastic reaction of the keratinocytes or angiogenic

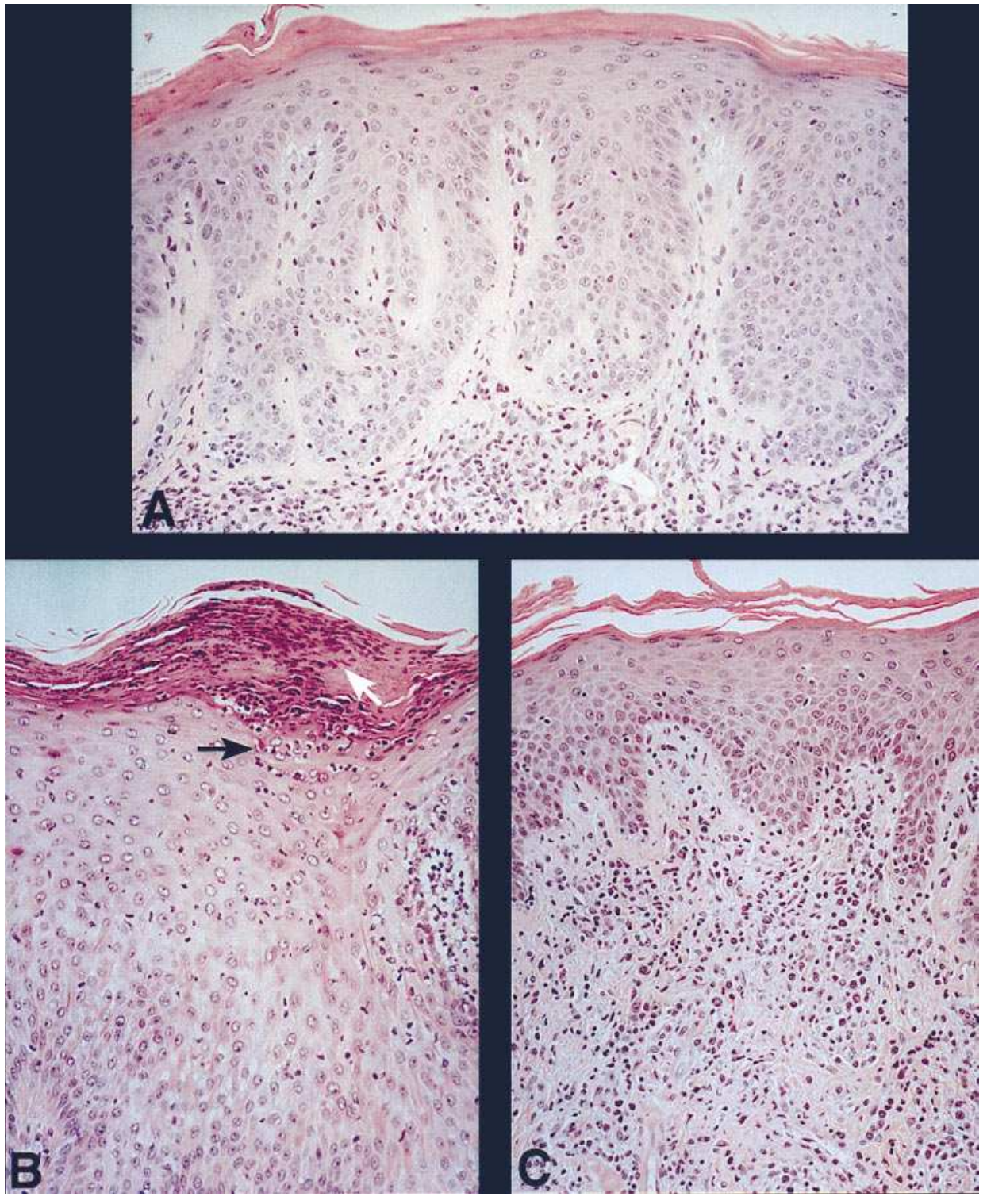

Figure 3. Histological features of transplanted PN skin injected with activated autologous immunocytes ( $A$ and $B$ ); and NN skin injected with activated autologous immunocytes $(C)$. In $A$, all the correct microscopic features of a psoriatic plaque are present in the injected skin grafts, making it indistinguishable from an idiopathic psoriatic plaque. In $B$, note the spongiform pustule of Kogoj (black arrow) and Munro's microabscess (white arrow). In $C$, injected NN skin has minimal scale, slight acanthosis, and mononuclear cells present in the epidermis and dermis. Hematoxylineosin skin; $A$ and $C, \times 100 ; B, \times 200$. 


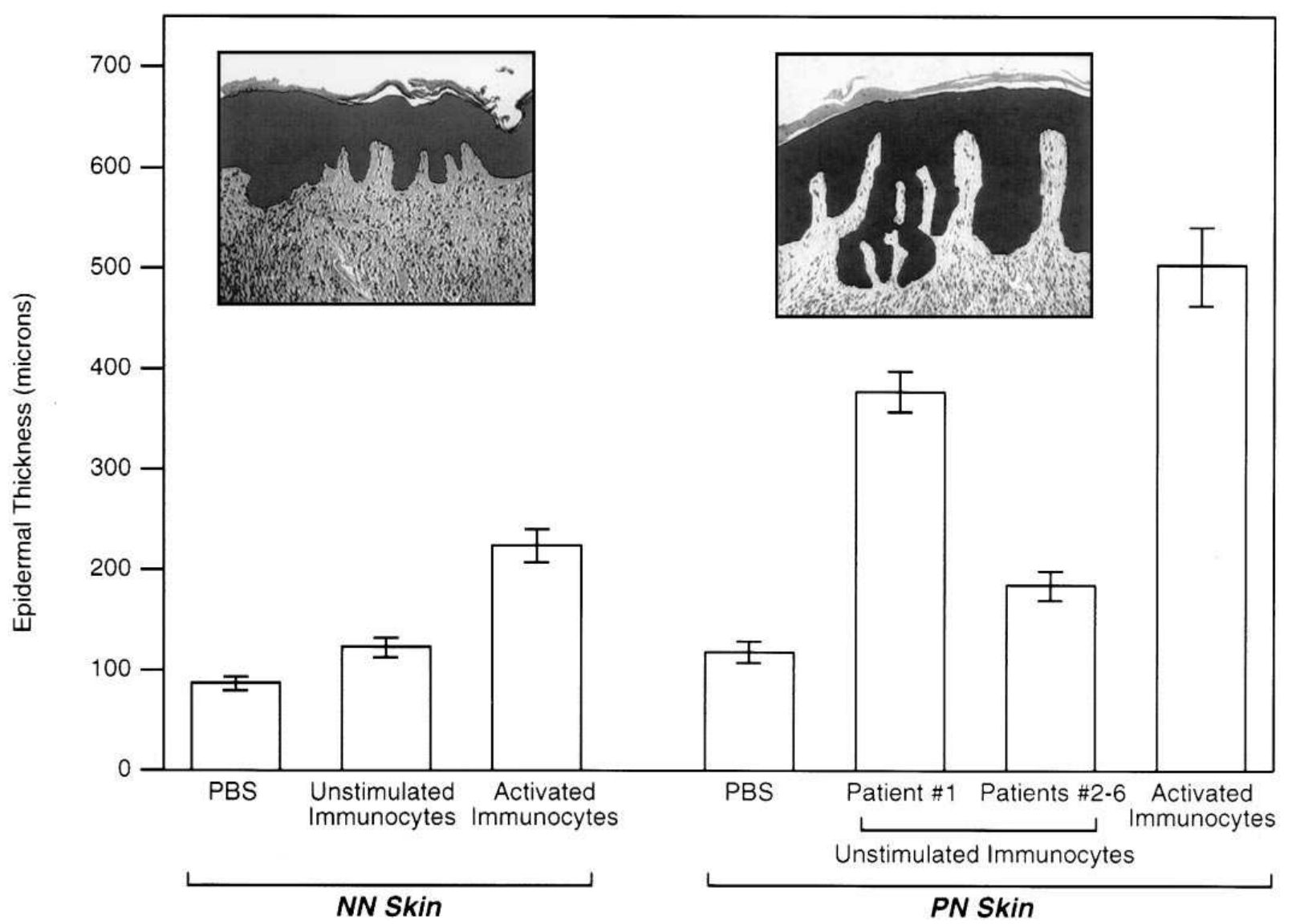

Figure 4. Epidermal thickness measurements for NN (left side) and PN (right side) skin following injection of indicated stimuli. The ordinate displays the mean epidermal thickness ( \pm standard error) determined by image analysis as described in Methods. The epidermal silhouette of representative NN and PN skin samples injected with activated autologous immunocytes is provided in the insets.

tissue reaction in the dermis as seen with psoriatic patientderived samples (Fig. $3 C$ ). The mean thickness measurements for NN skin are presented in Fig. 4 (left side) and reveal that after injection of unstimulated immunocytes or activated immunocytes the epidermis was $123 \pm 19$ and $224 \pm 33$ microns thick, respectively. Compared to NN skin injected with PBS, only when activated immunocytes were used did the increase in epidermal thickness have statistical significance $(P<0.05)$, although no full-fledged psoriatic plaques were produced.

Immunohistochemical analysis of transplanted human skin samples. To more fully characterize the response of PN skin to the various injected stimuli, immunophenotypic analysis was performed to detect a variety of relevant antigenic determinants. The extent of keratinocyte proliferation was assessed by immunostaining to detect proliferating cell nuclear antigen (PCNA). Compared to PN skin injected with PBS in which only approximately 1 out of every 10 keratinocytes in the basal cell layer had PCNA positive nuclei, in PN skin injected with activated immunocytes, approximately 1 out of every 3-4 keratinocytes in the basal cell layer were PCNA positive as well as numerous suprabasal keratinocytes. The specific number of PCNA positive keratinocytes observed for representative $1.3 \mu \mathrm{m}$ length segments of epidermis are as follows: $\mathrm{NN}$ skin + $\mathrm{PBS}=283 \pm 39 ; \mathrm{NN}$ skin + activated immunocytes $=721 \pm 93$; PN skin + PBS $=366 \pm 47$; PN skin + activated immunocytes $=2143 \pm 187$. Other indicators of keratinocyte activation that were measured are portrayed in Fig. 5, including HLADR, $\beta 1$ integrin expression and keratin 16 . For each of these antigens, PN skin injected with PBS revealed no expression by keratinocytes, but after injection by activated immunocytes, the epidermis became diffusely positive for HLA-DR (Fig. $5 A$ ), $\beta 1$ integrin (Fig. $5 B$ ), keratin 16 (Fig. $5 C$ ), and involucrin (data not shown) with patterns resembling those observed in idiopathic psoriatic plaques biopsied from untreated patients $(8,18,25,26)$. Mac 387 expression was present in PN skin injected with PBS by keratinocytes, but became more strongly and diffusely present following immunocyte injection (Fig. 5 D) (27).

To characterize the types of autologous immunocytes present in PN skin that had become phenotypically transformed into full-fledged psoriatic plaques, several additional immunohistochemical stains were performed. Human T cells infiltrating both dermis and epidermis were CD3 positive and CD45RO positive indicating that they belong to the "memory" type of lymphocyte subset, and they also expressed the IL-2 receptor indicating that they were activated (data not shown). As shown in representative profiles of grafts injected with activated immunocytes (Fig. 6) both epidermal and dermal compartments contained CD3, CD4 and CD8 positive $\mathrm{T}$ cells; although in all patients, there were more CD8 positive $\mathrm{T}$ cells in the epidermis compared to the CD4 positive $\mathrm{T}$ cell population. Infiltrating $\mathrm{T}$ cells were predominantly confined to 


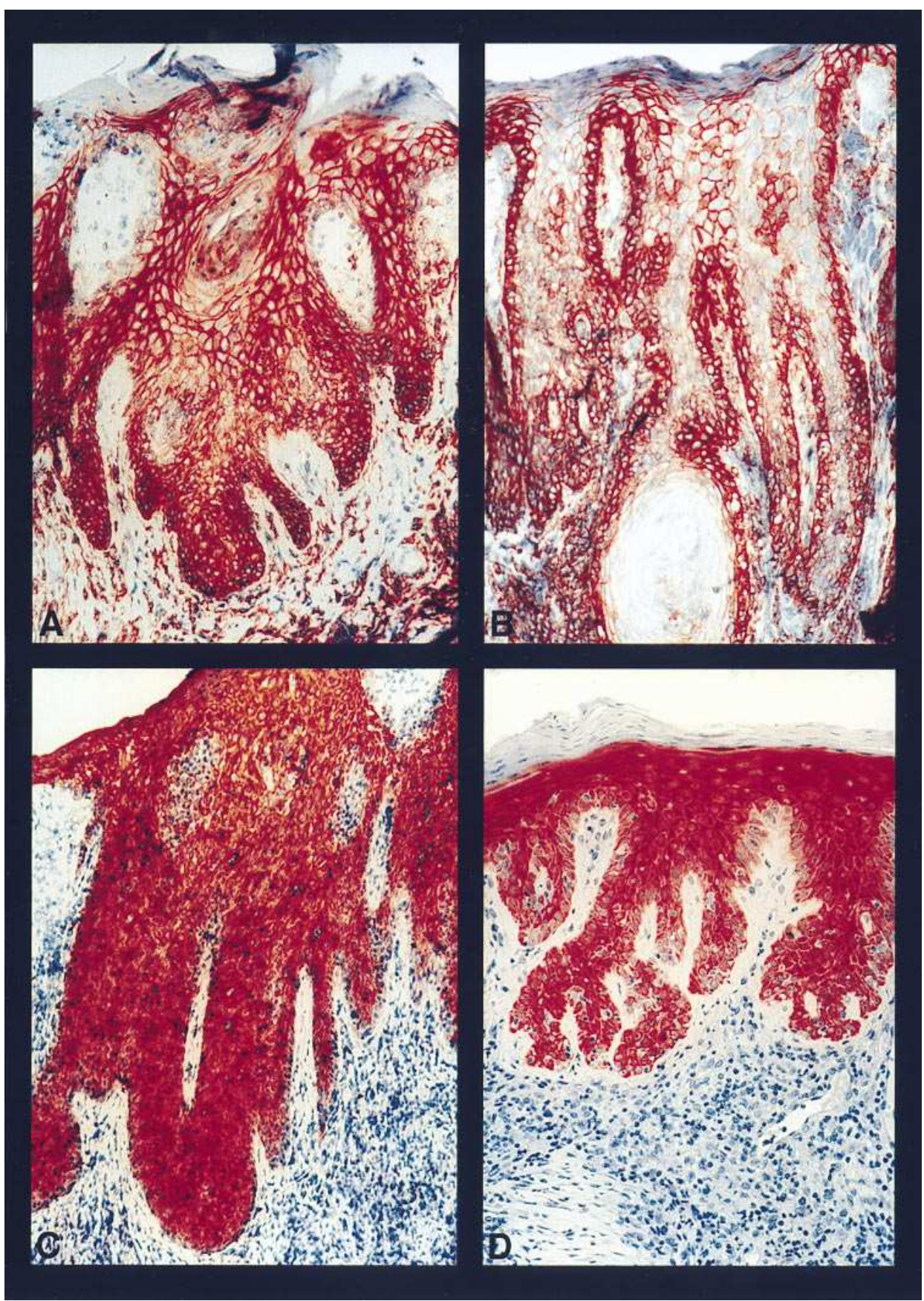

Figure 5. Immunohistochemical profile of PN skin injected with autologous activated immunocytes to detect: HLA-DR $(A)$; $\beta 1$ integrin $(B)$; keratin $16(C)$; MAC $387(D)$. For all of these antigens, note the strong and diffuse expression by the lesional keratinocytes $(A-D)$. Avidin-biotin immunoperoxidase stain. lower and mid-epidermal zones with a variable extent of infiltration amongst different grafts, and $\mathrm{T}$ cells displayed a nonrandom migration pattern after intradermal injection that was indistinguishable from idiopathic psoriatic plaques (28). There was also epidermal keratinocyte intercellular adhesion molecule-1 (ICAM-1) expression that accompanied the intra-epidermal T cells (data not shown) as previously described $(29,30)$. In terms of APCs, there were only rare scattered CD1a positive epidermal Langerhans cell in the epidermis, but there were numerous factor XIIIa positive dermal dendritic cells (data not shown). Once again, the number and pattern of factor XIIIa positive cells in the injected PN skin resembled naturally occurring psoriatic plaques $(30,31)$. Compared to the large number of factor XIIIa positive cells, there were only oc- casional CD14 positive macrophage-type cells in the dermal infiltrate (data not shown).

The expression of additional adhesion molecules that are relevant to the pathophysiology of psoriasis included $\mathrm{T}$ cells within the epidermis and dermis that expressed CLA, and the adjacent blood vessels had focal E-selectin, and diffuse vascular cell adhesion molecule-1 (VCAM-1) expression (data not shown), as previously described for untreated psoriatic plaques $(3,31)$.

\section{Discussion}

For several decades investigative skin biologists and clinicians have debated whether psoriasis is caused primarily by an ab- 


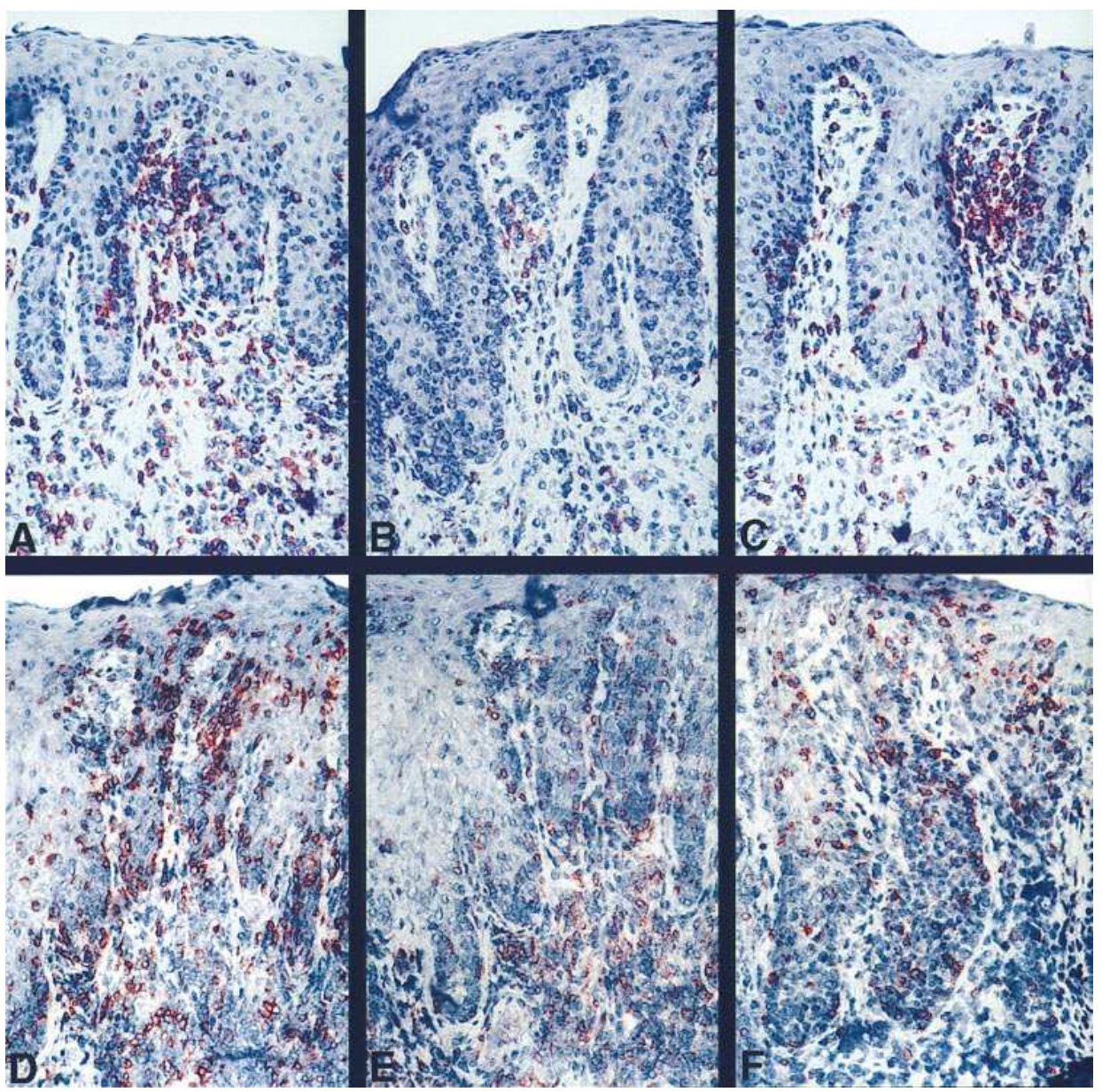

Figure 6. Distribution of CD3 ( $A$ and $D$ ); CD4 ( $B$ and $E$ ), and CD8 ( $C$ and $F)$ positive T cells in two different $P N$ skin grafts injected with autologous activated immunocytes. In $A-C$, the PN skin converted to PP skin is characterized by a $T$ cell infiltrate with relatively sparse $\mathrm{CD} 4$ positive cells in the dermis. In a different patient $(D-F)$, more $\mathrm{CD} 4$ positive $\mathrm{T}$ cells are present in the dermis. Also note that in both grafts, more $\mathrm{CD} 8$ positive $\mathrm{T}$ cells are present in the epidermis compared to CD4 positive T cells. Avidin-biotin immunoperoxidase stain. normality of the indigenous epidermal keratinocytes, or represents a disorder of the immune system whereby circulating exogenous disease producing immunocytes are recruited into the skin (3). By examining the epidemiology and genetics of psoriasis, it has not been possible to unequivocally identify a specific candidate molecule or cell type responsible for causing this common cutaneous disorder $(1,23,24,32,33)$. Likewise, numerous pharmacological studies, and several transgenic animal models have produced either inconclusive or conflicting results as to whether the keratinocyte or immunocyte is primarily at fault in psoriasis (5-19). In an attempt to identify the nature of the fundamental defect in psoriasis, we asked which cellular ingredients were necessary for full emergence of psoriatic lesions using the SCID-human skin chimeric animal model (20). The results were clear-cut in that activated autologous immunocytes obtained from blood could consistently convert PN skin into full-fledged psoriatic plaques. The expression of 20 different antigens (Table I) in skin converted from a PN to PP phenotype with patterns resembling idiopathic psoriatic plaques further validates the fidelity of this model system.

To the best of our knowledge, this complete phenotypic conversion of PN to PP skin is the first time that psoriatic lesions with all of the correct histological and immunohistochemical staining properties has been achieved. The induction of keratinocyte and endothelial cell activation cannot be considered as just a non-specific Koebner response, as the injection procedure itself using either several different soluble mediators (Table II), or the injection of non-activated immunocytes in 5 of 6 psoriatic patients, did not provoke this PN to $\mathrm{PP}$ conversion. Indeed in all but one of our psoriatic subjects, the blood-derived immunocytes required pre-activation for them to be able to produce such dramatic clinical and pathological changes in the injected PN skin. Such a pre-activation event of immunocytes may explain why patients given IFN- $\gamma$ or IL-2 develop psoriasis, and the inability of non-activated immunocytes to consistently produce full-fledged psoriatic plaques (Fig. 6) (34-36). It is also unlikely that injection of activated immunocytes produced a non-specific effect in skin, as there was no induction of psoriatic plaques or angiogenic tissue reactions in the $\mathrm{NN}$ skin samples. It will be of interest to determine if the correct recipe of both types and amounts of soluble mediators as described in Table II can be deciphered to produce full-fledged psoriaatic plaques using this experimental approach. To date we have not found the correct combination of cytokines or superantigens that in the absence of injected immunocytes provokes a complete phenotypic conversion of PN skin to PP skin. Thus, we conclude once activated, autologous immunocytes derived from the blood of psoriatic patients that are introduced into the dermis of PN skin, can appropriately traffic in a non-random fashion, and trigger keratinocyte and endothelial cell changes in the SCID model that are indistinguishable from idiopathic lesions that spontaneously develop in psoriatic patients. Perhaps the closest analogy that currently exists relative to our findings is a case report 
in which psoriasis was triggered after a bone marrow transplantation (37), and two case reports in which the psoriatic phenotype was cleared following bone marrow transplantation, and presumably elimination of disease-producing immunocytes from the general circulation $(38,39)$.

It should be noted that our transplantation-based results using SCID mice contradict earlier findings using nude mice that concluded there was an intrinsic defect in PN skin (40-44). In these studies, various non-immunological stimuli were used to produce an increase in epidermal thickness, but we do not believe that any of these published reports demonstrate fullfledged psoriasis. Using terms such as psoriasiform or psoriasis-like only tend to confuse the discussion, and we suggest that only full-fledged conversion to psoriatic plaques be considered as relevant to the dissection of the etiology and pathophysiology of this disease. Thus, we do not accept the previous conclusion that psoriatic skin can develop independently of circulating host factors (44). It is unclear at present whether the extremely thin and fragile nude mouse: human PN skin transplants can be converted to full fledged psoriatic plaques by injecting activated autologous immunocytes $(45,46)$. In any event, our current results using the SCID mouse transplant model demonstrate the requirement for participation of a cell type non-intrinsic to the skin (i.e., blood-derived activated immunocyte) for production of psoriatic plaques. However, it should be noted based on this current data we cannot exclude an additional defect in psoriatic keratinocytes that may contribute to the onset of plaque formation after intradermal injection of immunocytes. In ongoing studies, we have observed in 1 of $4 \mathrm{NN}$ skin samples injected with allogeneic immunocytes derived from a psoriatic patient, that psoriatic plaques developed within two weeks (our own unpublished observation) suggesting that even normal keratinocytes can sometimes be induced to express a psoriatic phenotype. There are several questions that can be raised and addressed in the future using this experimental model.

The most important question relates to the identity of the specific type of disease-producing $\mathrm{T}$ cell in psoriasis. Now that we have demonstrated that full-fledged psoriatic lesions can be produced reproducibly using this model, studies are under way to identify such pathogenic T cells. Our current results using a mixture of activated immunocytes do not provide any specific clues in this regard as the number of intraepidermal CD4 and CD8 positive cells was variable (Fig. 6), as is typically seen in idiopathic psoriatic plaques. Given recent findings by several groups of various clonal populations bearing specific (but not identical) $\mathrm{T}$ cell receptors in psoriatic lesions, a specific clone or group of specific T cell subsets may be isolatable (14, $17,47,48)$.

In conclusion, we believe the use of this SCID-human skin chimeric model represents a novel and valid experimental tool that can be relied on for future mechanistic studies into the pathophysiology and treatment of psoriasis. The present observations using this chimeric model system provide for the first time, direct evidence that immunocytes derived from blood can initiate the complete phenotypic transformation of PN skin into psoriatic plaques. While psoriasis is generally viewed as a skin disease because of the prominent clinical presentation of thick, scaling plaques, the current data strongly support a hypothesis advanced 10 years ago that suggested that psoriasis represents a pathological process driven primarily by cellular aberrations contained within the immune system (49). Using this model system, it should be possible to determine whether psoriasis represents an autoimmune disease, and the specific contributory role bacterial-derived superantigens play in its etiology (50).

\section{Acknowledgments}

We thank T. Ames for technical assistance, B. Bonish for the computer assisted image analysis, T. Hamilton for statistical evaluation, J. Voorhees for encouragement and support in procuring tissue samples, and C. Thompson for critical review of the manuscript.

Supported by National Institutes of Health grants AR38957, 40065, and 40488 (B.J. N). TW-S was supported by the Experimental Immunopathology Training Grant AI07413.

\section{References}

1. Farber, E.M., and M.L. Nall. 1974. The natural history of psoriasis in 5,600 patients. Dermatologica (Basel). 148:1-18.

2. Farber, E.M., B.J. Nickoloff, B. Recht, and J.E. Fraki. 1986. Stress, symmetry and psoriasis. J. Am. Acad. Dermatol. 14:305-311.

3. Nickoloff, B.J. 1991. The cytokine network in psoriasis. Arch. Dermatol. 127:871-884.

4. Nickoloff, B.J., L.A. Turka, R.S. Mitra, and F.O. Nestle. 1995. Direct and indirect control of $\mathrm{T}$ cell activation by keratinocytes. J. Invest. Dermatol. 105(Suppl.):25-29.

5. Baker, B.S., A.V. Powles, H. Valdimarsson, and L. Fry. 1988. An altered response by psoriatic keratinocytes to gamma interferon. Scand. J. Immunol. 28:735-740.

6. Nickoloff, B.J., R.S. Mitra, J.T. Elder, G.J. Fisher, and J.J. Voorhees. 1989. Decreased growth inhibition by recombinant gamma interferon is associated with increased production of transforming growth factor alpha in keratinocytes cultured from psoriatic lesions. Br. J. Dermatol. 121:161-174.

7. Nickoloff, B.J., R.S. Mitra, J. Varani, V.M. Dixit, and P.J. Polverini. 1994. Aberrant production of interleukin- 8 and thrombospondin-1 by psoriatic keratinocytes mediates angiogenesis. Am. J. Pathol. 144:820-828.

8. Carroll, J.M., M.R. Romero, and F.M. Watt. 1995. Suprabasal integrin expression in the epidermis of transgenic mice results in developmental defects and a phenotype resembling psoriasis. Cell. 83:957-968.

9. Baker, B.S., C.E.M. Griffiths, S. Lambert, A.V. Powles, H. Valdimarrson, and L. Fry. 1987. The effects of cyclosporin A on T lymphocyte and dendritic cell subpopulations in psoriasis. Br. J. Dermatol. 116:503-510.

10. Ho, V.C., C.E.M. Griffiths, C.E. Ellis, A.K. Gupta, C.C. McCuaig, B.J. Nickoloff, K.D. Cooper, and J.J. Voorhees. 1990. Intralesional cyclosporine A in the treatment of psoriasis. J. Am. Acad. Dermatol. 22:94-100.

11. Morel, P., J.P. Revillard, J.F. Nicolas, J. Wijdenes, H. Rizova, and J. Thivolet. 1992. Anti-CD4 monoclonal antibody therapy in severe psoriasis. $J$. Autoimmun. 5:465-477.

12. Breban, M., R.E. Hammer, J.A. Richardson, and J.D. Taurog. 1993. Transfer of the inflammatory disease of HLA-B27 transgenic rats by bone marrow engraftment. J. Exp. Med. 178:1607-1616.

13. Prinz, J.C., B. Gross, S. Vollmer, P. Trommler, I. Strobel, M. Meurer, and G. Plewig. 1994. T cell clones from psoriasis skin lesions can promote keratinocyte proliferation in vitro via secreted products. Eur. J. Immunol. 24:593-598.

14. Chang, J.C., L.R. Smith, K.J. Froning, B.J. Schwabe, J.A. Laxer, L.L. Caralli, H.H. Kurland, M.A. Karasek, D.I. Wilkinson, D.J. Carlo et al. 1994. $\mathrm{CD} 8+\mathrm{T}$ cells in psoriatic lesions preferentially use T-cell receptor $\mathrm{V}$ beta 3 and/or V beta 13.1 genes. Proc. Nat. Acad. Sci. USA. 91:9282-9286.

15. Nestle, F.O., L.A. Turka, and B.J. Nickoloff. 1994. Characterization of dermal dendritic cells in psoriasis. Autostimulation of T lymphocytes and induction of Th1 type cytokines. J. Clin. Invest. 94:202-209.

16. Bata-Csorgo, Z., C. Hammerberg, J.J. Voorhees, and K.D. Cooper. 1995. Kinetics and regulation of human keratinocyte stem cell growth in shortterm primary ex vivo culture. Cooperative growth factors from psoriatic lesional $\mathrm{T}$ lymphocytes stimulate proliferation among psoriatic uninvolved, but not normal, stem keratinocytes. J. Clin. Invest. 95:317-327.

17. Menssen, A., P. Trommler, S. Vollmer, D. Schendel, E. Albert, L. Gurtler, G. Riethmuller, and J.C. Prinz. 1995. Evidence for an antigen-specific cellular immune response in skin lesions of patients with psoriasis vulgaris. $J$. Immunol. 155:4078-4083.

18. Gottlieb, S.L., P. Gilleaudeau, R. Johnson, L. Estes, T.G. Woodworth, A.B. Gottlieb, and J.G. Krueger. 1995. Response of psoriasis to a lymphocyteselective toxin (DAB IL-2) suggests a primary immune, but not keratinocyte, pathogenic basis. Nat. Med. 1:442-447.

19. Boehncke, W.H., D. Dressel, T.M. Zollner, and R. Kaufmann. 1996. Pulling the trigger on psoriasis. Nature (Lond.). 379:777.

20. Nickoloff, B.J., S.L. Kunkel, M. Burdick, and R.M. Strieter. 1995. Hu- 
man psoriatic skin chimeras-validation of a new animal model. Am. J. Pathol. 146:580-588.

21. Leung, D.Y.M., M. Gately, A. Trumble, B. Ferguson-Darnell, P.M. Schlievert, and L.J. Picker. 1995. Bacterial superantigens induce T cell expression of the skin-selective homing receptor, the cutaneous lymphocyte-associated antigen, via stimulation of interleukin 12 production. J. Exp. Med. 181: $747-753$

22. Marrack, P., and J. Kappler. 1990. The staphylococcal enterotoxins and their relatives. Science (Wash. DC). 248:705-711.

23. Henseler, T., and E. Christophers. 1985. Psoriasis of early and late onset: characterization of two types of psoriasis vulgaris. J. Am. Acad. Dermatol. $13: 450-456$.

24. Krueger, G.G., and M. Duvic. 1994. Epidemiology of psoriasis: Clinical issues. J. Invest. Dermatol. 102(Suppl):145-183.

25. Gottlieb, A.B., B. Lifshitz, S.M. Fu, L. Staino-Coico, C.Y. Wang, and D.M. Carter. 1995. Expression of HLA-DR molecules by keratinocytes and presence of Langerhans cells in the dermal infiltrates of active psoriatic plaques. J. Exp. Med. 181:747-753.

26. Terui, T., S. Aiba, T. Kato, T. Tanaka, and H. Tagami. 1987. HLA-DR antigen expression on keratinocytes in highly inflamed parts of psoriatic lesions. Br. J. Dermatol. 116:87-93.

27. Kirkhana, N., S.J. Peacock, and D.B. Jones. 1990. Monoclonal antibody MAC387 recognizes a myelomonocytic antigen shared by epithelial cells in inflammatory skin diseases. Br. J. Dermatol. 122:61-69.

28. Nickoloff, B.J., and C.E.M. Griffiths. 1990. Lymphocyte trafficking in psoriasis: a new perspective emphasizing the dermal dendrocyte with active dermal recruitment mediated via endothelial cells followed by intra-epidermal T cell activation. J. Invest. Dermatol.(Suppl) 95:35-37.

29. Singer, K.H., D.T. Tuck, H.A. Sampson, and R.P. Hall. 1989. Epidermal keratinocytes express the intercellular adhesion molecule-1 in inflammatory dermatoses. J. Invest. Dermatol. 92:746-750.

30. Pankkoren, K., A. Naukkarinen, and M. Horsmanheimo. 1995. The development of manifest psoriatic lesions is linked with the appearance of ICAM-1 positivity on keratinocytes. Arch. Dermatol. Res. 287:165-170.

31. Cerio, R., C.E.M. Griffiths, K.D. Cooper, B.J. Nickoloff, and J.T. Headington. 1989. Characterization of factor XIIIa positive dermal dendritic cells in normal and inflamed skin. Br. J. Dermatol. 121:421-431.

32. Tomfohrde, J., A. Silverman, R. Barnes, M.A. Fernandez-Vinz, M. Young, D. Lory, L. Morris, K.D. Wuepper, P. Stastny, A. Menter, and A. Bowcock. 1994. Gene for familial psoriasis susceptibility mapped to distal end of human chromosome 17q. Science (Wash. DC). 264:1141-1145.

33. Nair, R.P., G. Sun-Wei, S. Jenisch, T.S. Henseler, E.M. Lange, M. Terhune, E. Westphal, E. Christophers, J.J. Voorhees, and J.T. Elder. 1995. Scanning chromosome 17 for psoriasis susceptibility: lack of evidence for a distal 17q locus. Hum. Hered. 45:219-230.

34. Fierlbeck, G., G. Rasner, and G. Muler. 1990. Psoriasis induced at injection site by recombinant gamma interferon. Arch. Dermatol. 126:351-355.

35. Lee, R.E., A.A. Gaspari, M.T. Lotze, A.E. Chang, and S.A. Rosenberg. 1988. Interleukin 2 and psoriasis. Arch. Dermatol. 124:1811-1815.
36. Boehncke, W.H., W. Sterry, A. Hainzl, W. Scheffold, and R. Kaufmann. 1994. Psoriasiform architecture of murine epidermis overlying human psoriatic dermis transplanted onto SCID mice. Arch. Dermatol. Res. 286:329-330.

37. Gardembas-Pain, M., N. Ifrah, C. Foussard, M. Boasson, and J.L. Verrat. 1990. Psoriasis after allogenic bone marrow transplantation. Arch. Dermatol. $126: 1523$.

38. Eedy, D.J., D. Burrows, J.M. Bridges, and F.G.C. Jones. 1990. Clearance of severe psoriasis after allogenic bone marrow transplantation. $\mathrm{Br}$. Med J. 300:908.

39. Jowitt, S.N., and J.A.L. Yin. 1990. Psoriasis and bone marrow transplantation. Br. Med. J. 300:1398-1399.

40. Krueger, G.G., D.D. Manning, J. Malouf, and B. Ogden. 1975. Longterm maintenance of psoriatic human skin on congenitally athymic (nude) mice. J. Invest. Dermatol. 64:307-312.

41. Briggaman, R.A., and C.E. Wheeler. 1980. Nude mouse-human skin graft model III. Studies on generalized psoriasis. J. Invest. Dermatol. 74:262 (Abstr.).

42. Haftek, M., J.P. Ortonne, M.J. Staquet, J. Viac, and J. Thivolet. 1981. Normal and psoriatic human skin grafts on "Nude" mice: morphological and immunochemical studies. J. Invest. Dermatol. 76:48-52.

43. Krueger, G.G., D.A. Chambers, and J. Shelby. 1981. Involved and uninvolved skin from psoriatic subjects: are they equally diseased? Assessment by skin transplanted to congenitally athymic (nude) mice. J. Clin. Invest. 68:15481557.

44. Fraki, J.E., R.A. Briggaman, and G.S. Lazarus. 1982. Uninvolved skin from psoriatic patients develops signs of involved psoriatic skin after being grafted onto nude mice. Science (Wash. DC). 215:685-687.

45. Reifenrath, W.G., E.M. Chellquist, E.A. Shipwash, W.W. Jederberg, and G.G. Krueger. 1984. Percutaneous penetration in the hairless dog, weanling pig, and grafted athymic nude mouse: evaluation of models for predicting skin penetration in man. Br. J. Dermatol. 111:123-135.

46. Baker, B.S., L. Brent, H. Valdimarsson, A.V. Powles, L. Al-Imara, M. Walker, and L. Fry. 1992. Is epidermal proliferation in psoriatic skin grafts on nude mice driven by T-cell derived cytokines? Br. J. Dermatol. 126:105-110

47. Lewis, H.M., B.S. Baker, S. Bokth, A.V. Powles, J.J. Garioch, H. Valdimarsson, and L. Fry. 1993. Restricted T-cell receptor V $\beta$ gene usage in the skin of patients with guttate and chronic plaque psoriasis. Br. J. Dermatol. 129: 514-520.

48. Leung, D.Y.M., J.B. Travers, R. Giorno, D.A. Norris, R. Skinner, J. Aelion, L.V. Kazemi, M.H. Kim, A.E. Trumble, M. Kotb, and P.M. Schlievert. 1995. Evidence for a streptococcal superantigen-driven process in acute guttate psoriasis. J. Clin. Invest. 96:2106-2112.

49. Valdimarsson, H., B.S. Baker, I. Jonsdottir, and L. Fry. 1986. Psoriasis: a disease of abnormal keratinocyte proliferation induced by T lymphocytes. $\mathrm{Im}$ munol. Today. 7:256-259.

50. Valdimarsson, H., B.S. Baker, I. Jonsdottir, A. Powles, and L. Fry. 1995. Psoriasis: a T-cell mediated autoimmune disease induced by streptococcal superantigens? Immunol. Today. 16:145-149. 